\title{
The scientific goal of the Japanese small astrometric satellite, Small-JASMINE
}

\author{
Taihei Yano, ${ }^{1}$ Naoteru Gouda, ${ }^{1}$ Yukiyasu Kobayashi, ${ }^{1}$ \\ Takuji Tsujimoto, ${ }^{1}$ Yoshito Niwa, ${ }^{1}$ and Yoshiyuki Yamada ${ }^{2}$ \\ ${ }^{1}$ National Astronomical Observatory, Mitaka, Tokyo, Japan \\ email: [yano.t, naoteru.gouda] @nao.ac.jp, yuki@merope.nao.ac.jp, [taku.tsujimoto, \\ kazin.niwa] @nao.ac.jp \\ ${ }^{2}$ Graduate School of Science, Kyoto University, Sakyo-ku, Kyoto 606-8502, Japan \\ email: yamada@scphys.kyoto-u.ac.jp
}

\begin{abstract}
Small-JASMINE is a small Japanese astrometric satellite, developed mainly at the National Astronomical Observatory of Japan. The target launch date of Small-JASMINE is around 2017. The satellite will be equipped with a telescope with an aperture size of $30 \mathrm{~cm}$ and a focal length of approximately $3.9 \mathrm{~m}$. The operational wavelength will be centered on the infrared $H w$ band, between 1.1 and $1.7 \mu \mathrm{m}$, using a $\mathrm{HgCdTe}$ detector with $4 \mathrm{k} \times 4 \mathrm{k}$ pixels. This will enable us to observe the central regions of our Galaxy and clarify the dynamical structure of the bulge region. A restricted region of the Galactic bulge will be observed using a frame-linking method, which is different from the approach taken by both Hipparcos and Gaia, both developed at ESA. The target accuracy of the annual parallax and proper motion is approximately $10 \mu$ as and $10 \mu$ as $\mathrm{yr}^{-1}$, respectively, in the central region of the survey area of $0.3 \times 0.3 \mathrm{deg}^{2}$. The target accuracy of the annual parallax, $\sim 50 \mu \mathrm{as}$, and that of the proper motion, $\sim 50 \mu$ as $\mathrm{yr}^{-1}$, will be obtained within a region of $2 \times 2 \mathrm{deg}^{2}$. The observing region covers a field of approximately $3 \times 3 \mathrm{deg}^{2}$. The mission is required to continue for around three years to obtain reliable measurements. In the winter season, the angular distance between the Sun and the Galactic bulge region is small. Accordingly, we may have the chance to observe different regions which contain scientifically interesting targets, such as Cygnus X-1. If we are successful in observing the object over the course of a few weeks, the orbital elements of the star accompanying Cygnus X-1 can be resolved by Small-JASMINE.
\end{abstract}

Keywords. astrometry, Galaxy: structure

\section{Introduction}

Future astrometric space missions, including Gaia and JASMINE (Japan Astrometry Satellite Mission for Infrared Exploration), will yield astrometric parameters - such as positions, parallaxes, and proper motions - of stars in the Galactic bulge. These satellites will produce astrometric data with an accuracy of $\sim 10 \mu$ as for parallaxes and $\sim 10 \mu$ as $\mathrm{yr}^{-1}$ for proper motions. As such, kinematic and dynamical information about Galactic bulge stars will be obtained after an observing period of order three years. Accordingly, it is expected that our understanding of the dynamical structure of the Galactic bulge will improve significantly.

\section{Specifications of Small-JASMINE}

The telescope of the Small-JASMINE astrometric satellite will have a primary-mirror diameter of $30 \mathrm{~cm}$, with a focal length of approximately $3.9 \mathrm{~m}$ (e.g., Yano et al. 2011). The optics layout of Small-JASMINE is shown in Fig. 1. The mission will use an observing 
Table 1. Specifications of Small-JASMINE

\begin{tabular}{lc}
\hline Optics & Modified Korsch $(3$ mirror $)$ \\
Diameter of primary mirror & $30 \mathrm{~cm}$ \\
Focal length & $3.9 \mathrm{~m}$ \\
Focal plane on the sky & $0.6 \times 0.6 \mathrm{deg}^{2}$ \\
Detector & $\mathrm{HgCdTe}$ \\
Target wavelength & $1.4 \mu \mathrm{m}(1.1 \leqslant \lambda \leqslant 1.7 \mu \mathrm{m})$ \\
Size of detector & $4 \times 4 \mathrm{~cm}^{2}(4 \mathrm{k} \times 4 \mathrm{k})$ \\
Pixel size & $10 \mu \mathrm{m}$ \\
Number of detectors & 1 \\
\hline
\end{tabular}
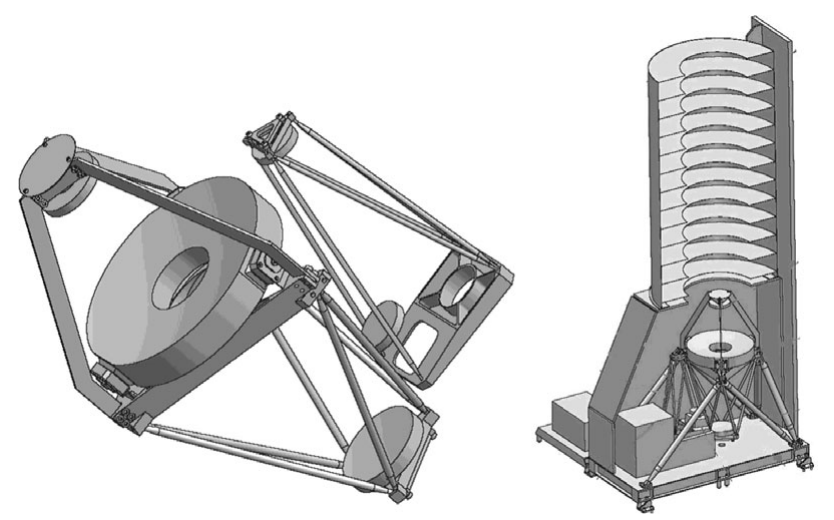

Figure 1. Small-JASMINE: Optics

wavelength centered on the infrared $H w$ band, between 1.1 and $1.7 \mu \mathrm{m}$ (and a central wavelength of $1.4 \mu \mathrm{m}$ ), using a $\mathrm{HgCdTe}$ detector. This will enable us to observe the central regions of our Galaxy and clarify the dynamical structure of the bulge region. The size of the detector is approximately $4 \times 4 \mathrm{~cm}^{2}$. The number of pixels is $4 \mathrm{k} \times 4 \mathrm{k}$ for a single detector, with a pixel size of $10 \times 10 \mu \mathrm{m}^{2}$. The focal plane of a single detector covers an area on the sky approximately $0.6 \times 0.6 \mathrm{deg}^{2}$. The target launch date of Small-JASMINE is around 2017. These parameters are summarized in Table 1.

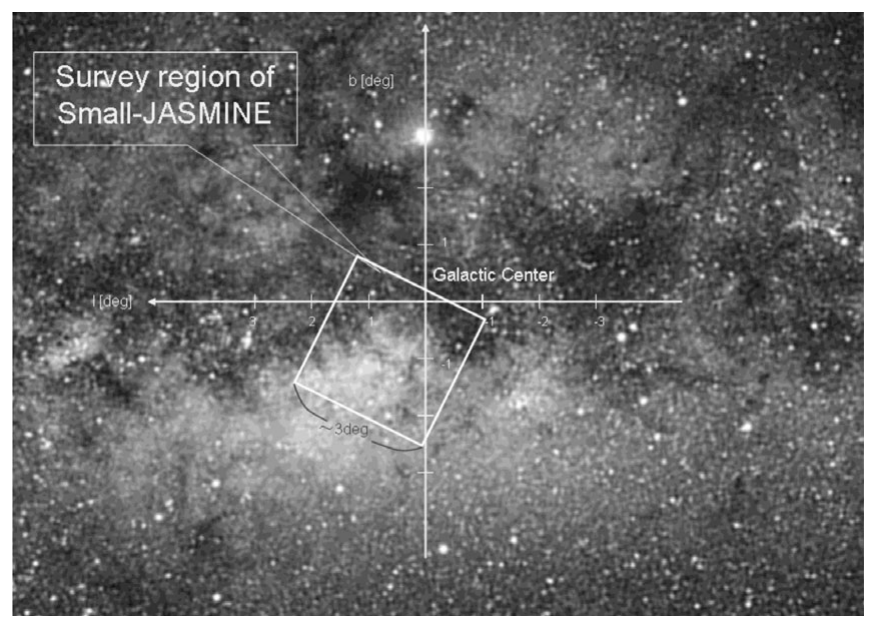

Figure 2. Survey region of Small-JASMINE 
Table 2. Accuracy for objects with $H w=11.5$ mag based on a three-year observing campaign.

\begin{tabular}{lcc}
\hline Parameter & $\begin{array}{c}\text { Accuracy for a region } \\
\text { of } 2 \times 2 \mathrm{deg}^{2}\end{array}$ & $\begin{array}{c}\text { Accuracy for the central } \\
\text { region }\left(0.3 \times 0.3 \mathrm{deg}^{2}\right)\end{array}$ \\
\hline Positional accuracy $\left(\sigma_{\text {pos }}\right)$ & $46 \mu \mathrm{as}$ & $9 \mu \mathrm{as}$ \\
Parallax accuracy $\left(\sigma_{\text {par }}\right)$ & $53 \mu \mathrm{as}$ & $11 \mu \mathrm{as}$ \\
Proper-motion accuracy $\left(\sigma_{\text {prop }}\right)$ & $53 \mu \mathrm{as} / \mathrm{yr}$ & $11 \mu \mathrm{as} \mathrm{yr}^{-1}$ \\
\hline
\end{tabular}

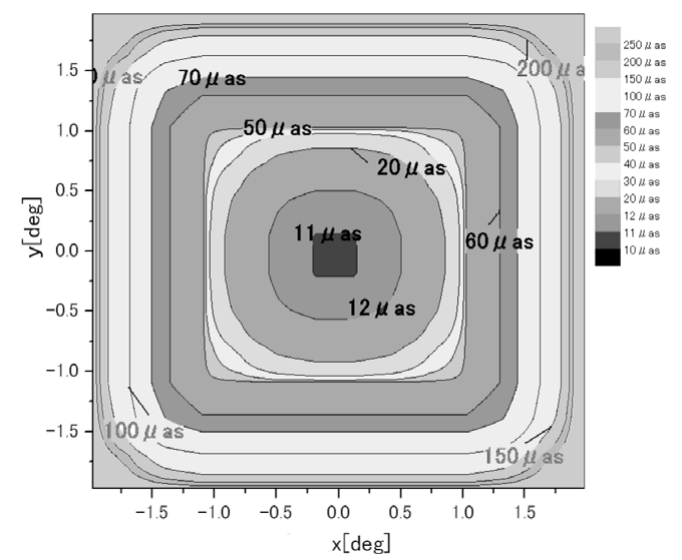

Figure 3. Expected accuracy of parallaxes for objects with $H w=11.5$ mag based on a three-year observing campaign.

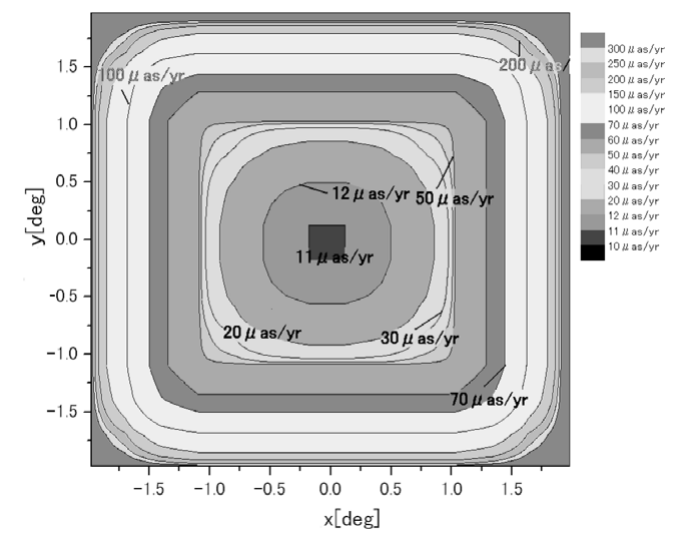

Figure 4. Expected accuracy of proper motions for objects with $H w=11.5$ mag based on a three-year observing campaign.

\section{Observing Region}

Small-JASMINE will observe the central regions of the Galactic bulge. The observations will produce astrometric parameters, including positions, parallaxes, and proper motions of stars. The Galactic bulge will be observed using a frame-linking method, which is different from the approach taken by both Hipparcos and Gaia, both developed at ESA. Observations using this method will cover roughly $3 \times 3 \mathrm{deg}^{2}$ of the central region of the Galactic bulge. The proposed region to be observed is shown in Fig. 2. 
Table 3. Accuracy for objects with $H w=11.5$ mag after one year of observations.

\begin{tabular}{lcc}
\hline Parameters & $\begin{array}{c}\text { Accuracy for a region } \\
\text { of } 2 \times 2 \mathrm{deg}^{2}\end{array}$ & $\begin{array}{c}\text { Accuracy for the central } \\
\text { region }\left(0.3 \times 0.3 \mathrm{deg}^{2}\right)\end{array}$ \\
\hline Positional accuracy $\left(\sigma_{\text {pos }}\right)$ & $79 \mu \mathrm{as}$ & $16 \mu \mathrm{as}$ \\
Parallax accuracy $\left(\sigma_{\text {par }}\right)$ & $140 \mu \mathrm{as}$ & $28 \mu \mathrm{as}$ \\
Proper-motion accuracy $\left(\sigma_{\text {prop }}\right)$ & $280 \mu \mathrm{as} / \mathrm{yr}$ & $55 \mu \mathrm{as} \mathrm{yr}^{-1}$ \\
\hline
\end{tabular}

Table 4. Accuracy for objects with $H w=10 \mathrm{mag}$ after one year of observations.

\begin{tabular}{lcc}
\hline Parameters & $\begin{array}{c}\text { Accuracy for a region } \\
\text { of } 2 \times 2 \mathrm{deg}^{2}\end{array}$ & $\begin{array}{c}\text { Accuracy for the central } \\
\text { region }\left(0.3 \times 0.3 \mathrm{deg}^{2}\right)\end{array}$ \\
\hline Positional accuracy $\left(\sigma_{\text {pos }}\right)$ & $40 \mu \mathrm{as}$ & $8 \mu \mathrm{as}$ \\
Parallax accuracy $\left(\sigma_{\text {par }}\right)$ & $71 \mu \mathrm{as}$ & $14 \mu \mathrm{as}$ \\
Proper-motion accuracy $\left(\sigma_{\text {prop }}\right)$ & $140 \mu \mathrm{as} / \mathrm{yr}$ & $23 \mu \mathrm{as}^{-1}$ \\
\hline
\end{tabular}

\section{Observing Accuracy}

Small-JASMINE will have a mission duration of 1-3 years. A three-year observing campaign will facilitate parallax and proper-motion accuracies of $\sim 10 \mu$ as and $\sim 10 \mu$ as $\mathrm{yr}^{-1}$, respectively, for objects with $H w=11.5 \mathrm{mag}$. The region for which these accuracies can be achieved is located in the center of the target observing region of $0.3 \times 0.3 \mathrm{deg}^{2}$. In addition, parallaxes with an accuracy of approximately $50 \mu$ as and proper motions of $\sim 50$ $\mu$ as $\mathrm{yr}^{-1}$ will be attained within a region of $2 \times 2 \mathrm{deg}^{2}$. These accuracies are summarized in Table 2 . The observing region covers a field of approximately $3 \times 3 \mathrm{deg}^{2}$. Accuracy maps for parallaxes and proper motions are shown in Figs 3 and 4, respectively. Based on the first year of observations, we expect to obtain parallaxes with an accuracy of $28 \mu$ as and

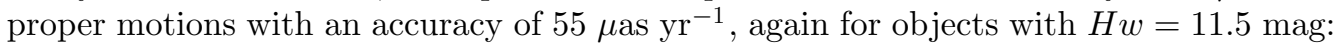
see Table 3 . In this case, we cannot derive distances to bulge stars with high accuracy. However, for objects with $H w=10 \mathrm{mag}$, we can obtain parallaxes and proper motions with accuracies of $14 \mu$ as and $23 \mu \mathrm{as} \mathrm{yr}^{-1}$ after one year: see Table 4 . In this case, we expect that we can obtain distances to bulge stars with high accuracy. Kinematic and dynamical information about Galactic bulge stars will be obtained after an observing period of roughly 1-3 years. Accordingly, it is expected that our understanding of the dynamical structure of the Galactic bulge will improve significantly.

\section{References}

Yano, T., Gouda, N., Kobayashi, Y., Tsujimoto, T., Hatsutori, Y., Murooka, J., Niwa, Y., \& Yamada, Y. 2011, in: Gaia: At the Frontiers of Astrometry (Turon, C., Meynadier, F., Arenou, F., \& Lindegren, L., eds), ESA Publ. Ser., 45, 449 